\title{
THE CLASS OF CO-NAMIOKA COMPACT SPACES IS STABLE UNDER PRODUCT
}

\author{
AHMED BOUZIAD
}

(Communicated by Franklin D. Tall)

\begin{abstract}
In an earlier paper we have established that the cartesian product of a family of co-Namioka compact spaces is co-Namioka if and only if all finite cartesian products of this family are co-Namioka. The purpose of this note is to show that the product of two co-Namioka compact spaces is always co-Namioka. The class of co-Namioka compact spaces is consequently stable under arbitrary products.
\end{abstract}

Let $X$ be a compact space. The space $X$ is said to be a co-Namioka compact space if, for any Baire space $B$ the following property $\mathcal{N}(B, X)$ is satisfied:

$\mathcal{N}(B, X)$ : For each separately continuous mapping $f: B \times X \rightarrow \mathbb{R}$ there is a residual set $A$ of $B$ such that $f$ is continuous at each point of $A \times X$.

This class of compact spaces, which was introduced by G. Debs in [3], has been extensively studied. (Cf. [4] and [5] for more information related to this topic.) In a previous paper [1], we have been interested in the study of the stability of this class under some topological operations; in particular, whether this class is stable under products. We have shown in [1, Théorème 2.2] that this class is stable under arbitrary products if and only if it is stable under finite products; and, concerning finite products, we have established the following [1, Théorème 2.1]: For every Valdiva compact space $X$ and for every co-Namioka compact space $Y$, the cartesian product $X \times Y$ is co-Namioka. Of course, these results set the problem of whether or not the product of two arbitrary co-Namioka compact spaces is co-Namioka. The aim of this note is to clarify this situation by giving a positive answer to this question. More precisely we establish the following somewhat stronger result:

Theorem 1. Let $X$ and $Y$ be two compact spaces and $B$ a Baire space. Suppose that $\mathcal{N}(B, X)$ and $\mathcal{N}(B, Y)$ are satisfied. Then the property $\mathcal{N}(B, X \times Y)$ is also satisfied.

Proof. Let $f: B \times(X \times Y) \rightarrow \mathbb{R}$ be a separately continuous mapping. Let $O \subset B$ be a nonvoid open set and $\varepsilon>0$. Let us show that there exists $b \in O$ such that the oscillation of $f$ is less than or equal to $\varepsilon$ at each point of $\{b\} \times(X \times Y)$. This will imply the theorem. Put $\eta=\varepsilon / 18$.

Fact 1 . There is a nonvoid open set $U \subset O$ and points $y_{1}, \ldots, y_{n}$ of $Y$ satisfying the following condition:

Received by the editors April 22, 1994 and, in revised form, October 1, 1994 1991 Mathematics Subject Classification. Primary 54C05, 54D30.

Key words and phrases. Namioka's property, separate continuity, continuity. 
For each $(b, y) \in U \times Y$ there exists $i \in\{1, \ldots, n\}$ such that

$$
\left|f(b, x, y)-f\left(b, x, y_{i}\right)\right| \leq \eta \quad \text { for all } x \in X .
$$

Assume that Fact 1 is false. We shall define a strategy $\sigma$ for player $\beta$ in the Choquet game on the space $O$ (see [2] or [6] for the definition of this game). Put $\sigma(\emptyset)=O$ and let $\left(x_{0}, y_{0}\right) \in X \times Y$. At the $n$-th stroke, if player $\alpha$ has played $V_{0}, \ldots, V_{n}$, then player $\beta$ chooses $b_{n} \in V_{n}, y_{n} \in Y$ and $x_{n, i} \in X, 0 \leq i<n$, such that

$$
\left|f\left(b_{n}, x_{n, i}, y_{n}\right)-f\left(b_{n}, x_{n, i}, y_{i}\right)\right|>\eta \quad \text { for all } 0 \leq i<n ;
$$

and then $\beta$ plays the nonvoid open subset $\sigma\left(V_{1}, \ldots, V_{n}\right)$ of $V_{n}$ given by

$$
\bigcap_{i<n}\left\{b \in V_{n} /\left|f\left(b, x_{n, i}, y_{n}\right)-f\left(b, x_{n, i}, y_{i}\right)\right|>\eta\right\} .
$$

As $O$ is a Baire space, $\sigma$ cannot be a winning strategy (cf. [6]); hence there is for $\alpha$ a winning game $\left(V_{n}\right)_{n \in \mathbb{N}}$ against $\sigma$. Let $b \in \bigcap_{n \in \mathbb{N}} V_{n}$. By the Stone-Weirstrass theorem there exist finitely many continuous real functions $g_{1}, \ldots, g_{k}$ defined on $X$ and $h_{1}, \ldots, h_{k}$ defined on $Y$, such that $\left|f(b, x, y)-\sum_{1}^{k} g_{i}(x) h_{i}(y)\right|<\eta / 3$ for every $(x, y) \in X \times Y$. Let $(\phi(n))_{n \in \mathbb{N}}$ be a subsequence of the natural numbers such that the sequences $\left(h_{i}\left(y_{\phi(n)}\right)\right)$ are convergent for all $i \in\{1, \ldots, k\}$. (Such a subsequence exists because all subspaces $h_{i}(Y)$ of $\mathbb{R}$ are compact.) Let $M \in \mathbb{R}_{+}^{*}$ such that $\left|g_{i}(x)\right| \leq M$ for each $i \in\{1, \ldots, k\}$ and for each $x \in X$. Take $n \in \mathbb{N}$ such that $\left|h_{i}\left(y_{\phi(n)}\right)-h_{i}\left(y_{\phi(n+1)}\right)\right| \leq \eta /(3 k M)$ for all $i \in\{1, \ldots, k\}$. It follows that

$$
\begin{aligned}
& \left|f\left(b, x_{\phi(n+1), \varphi(n)}, y_{\phi(n)}\right)-f\left(b, x_{\phi(n+1), \varphi(n)}, y_{\phi(n+1)}\right)\right| \\
& \quad \leq 2 \eta / 3+\left|\sum_{1}^{k} g_{i}\left(x_{\phi(n+1), \varphi(n)}\right)\left[h_{i}\left(y_{\phi(n+1)}\right)-h_{i}\left(y_{\phi(n)}\right)\right]\right| \\
& \quad \leq 2 \eta / 3+\eta / 3=\eta,
\end{aligned}
$$

which is a contradiction since $b$ belongs to $V_{\phi(n+2)}$. The proof of Fact 1 is complete.

It is clear that, similarly, we have the following:

Fact 2. For each nonempty open subset $O^{\prime}$ of $B$ there are a nonempty open set $V \subset O^{\prime}$ and points $x_{1}, \ldots, x_{m}$ of $X$ satisfying the following condition:

For each $(b, x) \in V \times X$ there exists $i \in\{1, \ldots, m\}$ such that

$$
\left|f(b, x, y)-f\left(b, x_{i}, y\right)\right| \leq \eta \quad \text { for all } y \in Y \text {. }
$$

Having established these two facts, to conclude the proof we shall combine them with the properties $\mathcal{N}(B, X)$ and $\mathcal{N}(B, Y)$. First, by applying $\mathcal{N}(B, X)$ to the separately continuous functions $(b, x) \in B \times X \rightarrow f\left(b, x, y_{i}\right) \in \mathbb{R}, i \in\{1, \ldots, n\}$, take a nonvoid open set $U_{1} \subset U$ so that the set

$$
W_{1}=\bigcap_{b \in U_{1}}\left(\bigcap_{i \leq n}\left\{\left(x, x^{\prime}\right) \in X \times X /\left|f\left(b, x, y_{i}\right)-f\left(b, x^{\prime}, y_{i}\right)\right|<\eta\right\}\right)
$$

is a neighborhood of the diagonal of $X \times X$. Now, let $U_{2} \subset U_{1}$ be a nonvoid open set and $x_{1}, \ldots, x_{m}$ be in $X$, satisfying Fact 2 . Finally, apply the property $\mathcal{N}(B, Y)$ to the functions $(b, y) \in B \times Y \rightarrow f\left(b, x_{i}, y\right) \in \mathbb{R}, i \in\{1, \ldots, m\}$, to get a nonvoid 
open set $U_{3} \subset U_{2}$ such that the set

$$
W_{2}=\bigcap_{b \in U_{3}}\left(\bigcap_{i \leq m}\left\{\left(y, y^{\prime}\right) \in Y \times Y /\left|f\left(b, x_{i}, y\right)-f\left(b, x_{i}, y^{\prime}\right)\right|<\eta\right\}\right)
$$

is a neighborhood of the diagonal of $Y \times Y$.

Let us now show that the oscillation of $f$ is less than or equal to $\varepsilon$ at each point of $U_{3} \times X \times Y$. Let $(b, x, y) \in U_{3} \times X \times Y$ and put $V_{1}=W_{1}[x]$ and $V_{2}=W_{2}[y]$. By Fact 1, we can fix an integer $j \in\{1, \ldots, n\}$ such that $\left|f(b, t, y)-f\left(b, t, y_{j}\right)\right| \leq \eta$ for all $t \in X$; let

$$
\begin{aligned}
V=U_{3} & \cap\left\{b^{\prime} \in B /\left|f\left(b^{\prime}, x, y\right)-f(b, x, y)\right|<\eta\right\} \\
& \cap\left\{b^{\prime} \in B /\left|f\left(b^{\prime}, x, y\right)-f\left(b^{\prime}, x, y_{j}\right)\right|<2 \eta\right\} \\
& \cap\left(\bigcap_{i \leq m}\left\{b^{\prime} \in B /\left|f\left(b^{\prime}, x_{i}, y_{j}\right)-f\left(b^{\prime}, x_{i}, y\right)\right|<2 \eta\right\}\right) .
\end{aligned}
$$

The set $V$ is a neighborhood of $b$ in $B$. Let $\left(b^{\prime}, x^{\prime}, y^{\prime}\right) \in V \times V_{1} \times V_{2}$. Since $b^{\prime} \in U_{2}$, there exists $i \in\{1, \ldots, n\}$ such that $\left|f\left(b^{\prime}, x^{\prime}, z\right)-f\left(b^{\prime}, x_{i}, z\right)\right| \leq \eta$ for each $z \in Y$; it follows that

$$
\begin{aligned}
\left|f(b, x, y)-f\left(b^{\prime}, x^{\prime}, y^{\prime}\right)\right|<\mid & f(b, x, y)-f\left(b^{\prime}, x, y\right) \mid \\
& +\left|f\left(b^{\prime}, x, y\right)-f\left(b^{\prime}, x, y_{j}\right)\right| \\
& +\left|f\left(b^{\prime}, x, y_{j}\right)-f\left(b^{\prime}, x^{\prime}, y_{j}\right)\right| \\
& +\left|f\left(b^{\prime}, x^{\prime}, y_{j}\right)-f\left(b^{\prime}, x_{i}, y_{j}\right)\right| \\
& +\left|f\left(b^{\prime}, x_{i}, y_{j}\right)-f\left(b^{\prime}, x_{i}, y\right)\right| \\
& +\left|f\left(b^{\prime}, x_{i}, y\right)-f\left(b^{\prime}, x_{i}, y^{\prime}\right)\right| \\
& +\left|f\left(b^{\prime}, x_{i}, y^{\prime}\right)-f\left(b^{\prime}, x^{\prime}, y^{\prime}\right)\right| \\
\leq & 9 \eta=\varepsilon / 2 .
\end{aligned}
$$

This shows that the oscillation of $f$ at $(b, x, y)$ is less than or equal to $\varepsilon$.

A consequence of Theorem 1 is that the product of two (hence finitely many) co-Namioka compact spaces is co-Namioka. From [1, Théorème 2.2] we then get the stability result mentioned in the title:

Theorem 2. The class of co-Namioka compact spaces is stable under arbitrary product.

\section{ACKNOWLEDGMENT}

I am indebted to G. Debs and to an anonymous referee for their observations concerning the proof of Fact 1 .

\section{REFERENCES}

1. A. Bouziad, Notes sur la propriété de Namioka, Trans. Amer. Math. Soc. 344 (1994), 873-883. MR 94m:54032

2. G. Choquet, Lectures on analysis I, Benjamin, New York, 1969. MR 40:3252

3. G. Debs, Points de continuité d'une application séparément continue, Proc. Amer. Math. Soc. 97 (1986), 218-228. MR 87c:54014 
4. S. Merkourakis and S. Negrepontis, Banach spaces and topology II, Recent Progress in General Topology (M. Hušek and J. van Mill, eds), Elsevier Sciences Publishers, Amsterdam, 1992, pp. 495-536. CMP $93: 15$

5. D. B. Shakmatov, Compact spaces and their generalizations, Recent Progress in General Topology (M. Hušek and J. van Mill, eds.), Elsevier Sciences Publishers, Amsterdam, 1992, pp. 571-640.

6. J. Saint Raymond, Jeux topologiques et espaces de Namioka, Proc. Amer. Math. Soc. 87 (1983), 499-504 MR 83m:54060

Université de Rouen, U. F. R. Des Sciences, URA C. N. R. S. D 1378, 76821 Mont Saint Aignan Cedex, France

E-mail address: ahmed.bouziad@univ-rouen.fr 